\title{
Voluntarios deportivos versus profesionales remunerados: dificultades y conflictos del desempeño dela acción voluntaria en la organización de acontecimientos deportivos Sport volunteers versus remunerated professionals: difficulties and conflicts
of the performance of the voluntary action in the organization of sport events
}

\author{
Inmaculada Pérez Pérez* y María Elena García Montes** \\ * Colegio Claret de Sevilla y ** Universidad de Murcia
}

\begin{abstract}
Resumen: Detectar los inconvenientes que puede encontrar una persona para formar parte de un grupo de voluntariado deportivo es el objetivo planteado, discriminando y contrastando, para ello, entre las opiniones del propio voluntariado deportivo y las del personal profesional remunerado que ha coordinado a grupos de voluntariado. La investigación se ha desarrollado, por un lado, mediante la aplicación de un cuestionario autoadministrado al total de población (251) asistente a los «Cursos de formación para Voluntarios Deportivos de Andalucía», realizados en las diferentes provincias andaluzas en el año 2002, y, por otro lado, a través de otro cuestionario para los profesionales remunerados que trabajan con voluntariado, utilizando un muestreo no aleatorio opinático, a un total de 50 sujetos. Los resultados han puesto de manifiesto la mayor percepción de inconvenientes por parte del personal profesional remunerado, que el propio voluntariado deportivo, sobre las implicaciones de la labor a desarrollar por estos últimos. El voluntariado destaca sobretodo el compromiso temporal que esto supone, mientras que los profesionales hacen mayor insistencia en los aspectos relacionados con la propia organización.
\end{abstract}

Palabras clave: Aventura, experiencia, educación, juego y naturaleza.

\begin{abstract}
To detect the disadvantages that can find a person to be member of a group of sport voluntary is the raised objective, discriminating and contrasting, for it, between the opinions of the own sport voluntary service and those of the remunerated professional personnel who has coordinated voluntary service groups. The investigation has been developed, on the one hand, by means of the application of a self-administrated questionnaire to the total of population (251) attending to the «Courses of formation for Sport Volunteers in Andalusia», made in the different andalusian provinces in 2002, and, on the other hand, through another questionnaire for the remunerated professionals who work with voluntary service, using a nonrandom sampling opinático, to a total of 50 subjects. The results have shown the greater perception of disadvantages on part of the remunerated professional personnel, who the own sport voluntary service, on the implications of the work to develop at these last ones. The voluntary service emphasizes coverall the temporary commitment that this supposes, whereas the professionals make greater insistence in the aspects related to the own organization.
\end{abstract}

Key words: Sport events. Volunteer service. Participation. Obstacles.

\section{Introducción.}

Cuando hablamos de actuaciones voluntarias siempre aparecen dos elementos básicos definidos claramente por las distintas leyes sobre el voluntariado que existen, se trata de las personas que deciden actuar como voluntarias y la organización que gestiona dicha intervención. Entre ellas, a diferencia de las actividades de tipo profesionalremunerada, no existe un contrato calculador (valora la media entre el esfuerzo desarrollado y la futura compensación), sino más bien un contrato denominado «psicológico» o «cooperativo» (Handy, 1988).

Dicho «contrato» aparece cuando una persona decide vincularse a entidades con las que se comparte objetivos e intereses, sin esperar nada a cambio, por lo que en la medida que esto se mantenga en el tiempo, la relación también se alargará. Este enfoque nos da a entender que la base de este vínculo parte del altruismo y desinterés de las personas voluntarias, todo ello teniendo siempre presente el derecho de los colaboradores a retirar su participación, si fuera necesario.

Aunque también se podría plantear una relación de tipo «coercitivo»» en el mundo de las actuaciones voluntarias. Esta situación negativa entre la entidad y voluntario, es el resultado de una realidad en las que los voluntarios se sienten obligados a seguir vinculados directamente con la organización, teniendo la sensación de que si no se mantienen implicados la institución se vendría abajo. Esto se da con cierta frecuencia en aquellas personas que actúan en el campo del Voluntariado Deportivo, trabajando con grupos de jóvenes en diferentes deportes. Monitores o entrenadores que comienzan su andadura con las organizaciones de

Fecha de recepción: 05-08-06 - Fecha de aceptación: 03-10-06

Correspondencia: María Elena García Montes

C/Cabo Vidio, 27

30730 San Javier - Murci

E-mail: garciamo@um.es manera cooperativa, pero alcanzan un determinado momento en que la situación se vuelve coercitiva, por falta de tiempo, de variaciones de las motivaciones o intereses, cambios en la vida personal o profesional...

En lo que respecta al deporte, más cncretamente a los grandes acontecimientos deportivos, reflejar, tal y como expone Heinemann (2004, p. 11), «que se ha convertido en una compleja industria en el marco de un sistema económico de mercado libre como una red poco diáfana de interrelaciones industriales basadas en una gran diversidad de intereses que requieren para su conjunción negociaciones en varios niveles»

Ante lo expuesto anteriormente se puede decir que el sector voluntario no está libre de presiones, a pesar de la buena voluntad de las personas colaboradoras y del entorno positivo en el que se debe producir estas actuaciones solidarias. Se encuentran inconvenientes de todo tipo, relacionados con los propios voluntarios como personas, con las organizaciones a las que se vinculan y con el entorno que rodea a este mundo (Domingo, 1996; Aguilera Luna, 2003; Cortés García y Matías Cabrera, 2004)

Resulta ser un problema importante el escaso tiempo que sobra tras la realización de la tareas obligatorias diarias, ya sean desde el punto de vista profesional (trabajo remunerado) o personal (familia, amigos, otras actividades de ocio...). Aunque se puede entender también esta escasez de tiempo disponible para la realización de tareas voluntarias, como resultado de la mayor gama de oportunidades para la utilización del ocio (Robinson y Godbey, 1999; VV.AA., 2004b).

Aparece como obstáculo del sector voluntario en relación a las organizaciones de esta índole, la necesidad cada vez mayor de realizar tareas más parecidas a las que se realizan en el sector profesional (público o privado), exigiendo la propia institución una preparación previa o una formación específica para poder colaborar como voluntario. Esta realidad hace que los propios voluntarios se sientan preocupados con el aumento de las exigencias referente a sus destrezas, pudiendo ser esto 
una circunstancia utilizada como excusa para la no realización y abandono de las actuaciones voluntarias.

En cuanto a los inconvenientes del sector voluntario en relación con la sociedad y el entorno en el se producen, se podría inicialmente considerar la redefinición del término ocio como un elemento materialista que se compra y vende (Weisbrod, 1978), empezando a formar parte de la sociedad consumista actualmente predominante. Así cuando se realizan actuaciones voluntarias en cualquier campo, se busca el tener contento a las personas que reciben directamente los beneficios de dichas actuaciones, olvidando al propio ejecutante de dicha acción y por ende la base de este movimiento social y cívico. Esta falta de reconocimiento hacia los voluntarios y sus actuaciones llega ser considerado un obstáculo que puede llegar a mermar las motivaciones e inquietudes de los colaboradores (Nichols y King, 1998; Heinemann, 1999).

Por otra parte podemos identificar otras características negativas del trabajo voluntario organizado que perjudican su desarrollo y expansión. Entre ellas se presenta una división del trabajo poco rígida y poco respetada que origina a menudo una superabundacia de nuevos roles (sobretodo de coordinación) y una escasez de otros (en particular de trabajos auxiliares) sufriendo los propios sujetos una tendencia a la autoinfravaloración que puede llegar a provocar riesgos de abandono (Collozi y Bassi, 1995).

Pero todos estos caracteres que van en detrimento de la profesionalidad y de la eficacia, influyen de forma muy marginal sobre la solidez de la acción voluntaria y sobre la eficacia de sus actuaciones. Todo ello gracias a la fuerza de las relaciones humanas sostenidas no sobre vínculos contractuales, sino sobre la convicción de un proyecto común basado en la solidaridad, alrededor de un campo importante en sus vidas y en su entorno más inmediato como es en este caso el deporte.

Otro límite que a menudo se ha convertido en un verdadero «tabú» para las organizaciones de voluntariado es la relación que existe entre el trabajo profesional y el voluntario, considerados incompatibles, en base al teorema «profesionalidad» igual a «remuneración» y distinto de «acción voluntaria». Esto se ve agravado por la diversidad de titulaciones, heterogeneidad de perfiles de los profesionales implicados en la coordinación de este tipo de actuaciones y la consecuente falta de definición de las responsabilidades de unos y otros (Roca, 2003). Sin embargo nos damos cuenta que precisamente la combinación del trabajo profesionalvoluntario, ha permitido alcanzar actividades mixtas de manera exitosa llegando a realizar actuaciones que de ninguna otra manera hubieran salido a flote con trabajos realizados exclusivamente por voluntarios o por profesionales remunerados (Domingo, 1996; Cortés, Hernán y López, 1997; VV.AA., 2004a).

Según se ve reflejado en el Plan General del Deporte en Andalucía (VV.AA., 2004a: 252) «la labor del voluntario en el seno de una organización, puede ser de colaboración con el trabajado profesional, puede ser una labor complementaria sin llegar a sustituirla y, en su caso, bajo la dirección y supervisión; e incluso en ocasiones, puede ser una labor suplementaria, cuando aquella prestación sea adicional a la de los servicios públicos. Sin embargo, en ningún caso es posible una plena equiparación de tareas entre voluntarios y el trabajador profesional».

Hay que pensar que el trabajo profesional organiza el voluntario y su actividad solidaria y, a su vez, el voluntario es directa o indirectamente-según el tipo de entidad- el que da sentido a la actividad profesional. Es más, la mayoría de los profesionales son al mismo tiempo voluntarios, del mismo modo que muchos voluntarios se convertirán con el tiempo en profesionales a través de un proceso de inserción laboral (Abadía, 2004; Chavarria, 2005; Vaca, 2002). Al final se trata de un campo motivacional que recorre un continuo en el que puede haber diferentes tipos de prácticas sociales que conviven buscando un último objetivo común basado en la solidaridad (Martínez del Castillo, 1997; Marbán Gallego y Rodríguez Cabrero, 2001).

De esta manera, la figura del voluntariado no es algo a lo que se deba acudir como recurso aislado, sino que siempre debe ir unido al trabajo o actividad del personal profesional remunerado. Esta relación obligatoria y a la veznecesaria en muchas ocasiones no tiene que plantearse como oposición o suma de trabajos, sino como complementariedad de acciones para el correcto desempeño del trabajo asignado.

\section{Metodología.}

El uso extensivo del Voluntariado Deportivo en grandes acontecimientos, produce en muchas ocasiones conflictos no sólo entre el sector de profesionales que ven usurpadas sus posibles competencias en el terreno laboral por personas «no cualificadas», sino igualmente entre los propios Voluntarios y la organización que pone en marcha el evento. Detectar estas situaciones es uno de los objetivos principales de la investigación sobre el Voluntariado Deportivo que se ha llevado a cabo en la Comunidad Andaluza (Pérez Pérez, 2004). Para ello se han tenido en cuenta las opiniones tanto del Voluntariado Deportivo, como las del personal profesional remunerado que ha coordinado a grupos de voluntariado, con objeto de conocer las opiniones de uno y otro colectivo y poder contrastarlas.

Optamos por emplear el método descriptivo. Para obtener esta información acerca de datos que se han producido en el pasado y que forman parte de las experiencias personales, no se puede emplear la observación directa, sino que recurrimos al informe proporcionado por los propios Voluntarios Deportivos y por los profesionales remunerados, decidiéndonos por el estudio tipo encuesta o «survey», en palabras de Colás (1994).

La posibilidad de reunir a Voluntarios Deportivos se presenta a partir de la celebración de algún acontecimiento deportivo de relevancia. Esta circunstancia nos llevó a acceder a un grupo estable de Voluntarios Deportivos, denominado «Voluntarios Deportivos de Andalucía» (pertenecientes a la Consejería de Turismo y Deporte de la Junta de Andalucía), que van a tener opción de intervenir en cualquier evento deportivo dentro del territorio andaluz. El colectivo al que nos referimos lo forman todos los sujetos participantes en los «Cursos de formación para Voluntarios Deportivos de Andalucía» desarrollados por la Junta de Andalucía, a través del Instituto Andaluz del Deporte. Estos encuentros se llevaron a cabo a lo largo del año 2002, con un total de diez cursos repartidos por todas las provincias de la Comunidad Autónoma Andaluza, de manera gratuita, con aforo ilimitado y sin ningún requisito previo. Cumpliendo la organización con la obligación marcada por la ley $\left(\mathrm{BOJAn}^{\circ} 84,2001\right)$ de formar a todas las personas que deseen colaborar como Voluntario Deportivo.

A ellos se inscribieron sujetos que ya habían colaborado en algún momento como Voluntario Deportivo, al igual que otros para quienes el primer contacto que tenían con este tipo de actividades se producía a través de estos cursos de formación. Fueron en total 251 las personas inscritas y que finalizaron correctamente dichos cursos de preparación, pudiéndose realizar de manera organizada, rápida y estable la pasación autoadministrada por grupos del cuestionario y la recogida de datos.

Por otra parte, aparece la misma problemática a la hora de acceder a la población de profesionales remunerados que trabajan con Voluntarios Deportivos, ya que no existe oficialmente esta figura en muchas de las administraciones locales. Son los trabajadores de las distintas concejalías o patronatos de deportes de los ayuntamientos los que, en un determinado momento, dentro de sus puestos de trabajo habituales (técnicos deportivos, coordinadores deportivos...), se hacen cargo de un grupo de sujetos que voluntariamente quieren ayudar a organizar y poner en marcha un determinado acontecimiento deportivo. Sólo en la Junta de Andalucía, en la Consejería de Turismo y Deporte, aparece un departamento en el que existe de manera oficial personal cualificado para captar, coordinar y trabajar con Voluntarios Deportivos.

Para este segundo grupo estudiado, el del profesional remunerado, hemos utilizado un muestreo no aleatorio opinático. En este caso, se seleccionó una muestra, siguiendo los criterios establecidos en la investigación, de tal forma, que ésta es la más representativa a los efectos del estudio que se estaba realizando, según la experiencia propia del grupo investigador y que se ajustaban perfectamente al problema objeto de la investigación. 
Según esto, se escogió como muestra a sujetos que encontramos en la Empresa Pública Deporte Andaluz S.A, concretamente en el departamento de Eventos Deportivos que habían trabajado con grupos de Voluntarios Deportivos en distintos eventos organizados por la Consejería de Turismo y Deporte de la Junta de Andalucía. Igualmente, se acudió al área de deportes de algunos Ayuntamientos de la Comunidad Andaluza en los cuales se tenía conocimiento de que se había utilizado el recurso del Voluntariado Deportivo para organizar algún acontecimiento deportivo. En total se recogieron 50 cuestionarios, autoadministrados individualmente, de sujetos pertenecientes al colectivo del personal profesional remunerado que trabaja con Voluntarios Deportivos.

Se han empleado técnicas de análisis cuantitativo, codificando los datos recogidos para su tratamiento estadístico. Éstos, tabulados y mecanizados informáticamente, fueron analizados mediante al paquete de programas informático SPSS para Windows, v. 11.1. Para el análisis descriptivo se han utilizado las tablas de distribución de frecuencias y el análisis inferencial se ha desarrollado mediante la prueba del chi cuadrado para constatar el grado de asociación/relación entre variables.

\section{Análisis y discusión de resultados.}

3.1. Inconvenientes para colaborar como Voluntario Deportivo, según la opinión de los propios voluntarios.

El Voluntariado Deportivo, a la hora de percibir los inconvenientes de la acción voluntaria, corrobora una gran variedad de elementos que pueden distorsionar estas actuaciones y que podrían conllevar al abandono de este colectivo, aunque sólo uno de ellos está confirmado por más de la mitad de los sujetos y, en la mayoría de los casos, puede ser debido a los cambios personales y profesionales que sufren las personas y el no poder controlar el tiempo dedicado a cada parcela de sus vidas. Así el compromiso en cuanto al tiempo empleado en las actividades voluntarias (52.6\%) es el máximo inconveniente para poder actuar o no como tales, seguido a distancia, aunque relacionado también con la disponibilidad temporal, por su repercusión frente a otras actividades de tiempo libre (33.9\%) que tienen que abandonar en un momento determinado (figura 1).

Los siguientes inconvenientes tienen que ver con la organización y no con el trabajo voluntario directamente: el poco reconocimiento y valor oficial (33.5\%), que la organización no sea la correcta $(28.3 \%)$, que el trabajo asignado no sea el esperado y desilusione (21.9\%), que exista falta de claridad en la funciones y responsabilidades (21.5\%), que los objetivos y motivaciones personales no se adapten a los de la organización (19.9\%) o que se produzca una mala consideración por parte del personal remunerado (15.1\%). Todos ellos son motivos suficientes para que el voluntariado se plantee abandonar la organización y por tanto el trabajo voluntario.

Lo que si aparece claro es que a muy pocos de los voluntarios les preocupa tener que trabajar en equipo (8.8\%), si las dietas son o no correctas, o el gasto económico que puede llegar a suponer este tipo de actividades $(9.2 \%)$. Igualmente apenas se plantean sus colaboraciones como un cúmulo de derechos, deberes y responsabilidades que este tipo de trabajo conlleva (10\%). Todo ello nos lleva a pensar que los temores e inconvenientes más importantes indicados por los voluntarios deportivos parten de actuaciones desde el exterior y no desde su propia iniciativa de actuar voluntaria y solidariamente.

Para este tipo de personas que se acercan al Voluntariado Deportivo, no llega a suponer un obstáculo que afecte a sus relaciones profesionales (13.5\%), familiares (5.6\%) o a las amistades (4\%). Al contrario, ya que muchos de ellos se acercan a su campo profesional actual o futuro desde otra perspectiva y con otras sensaciones diferentes a las de un diario. Surge además por otra parte la posibilidad inmediata de conocer y hacer nuevas amistades.

Aparece un cierto reparo, aunque no muy determinante, que podría llegar a ser un inconveniente para el $13.9 \%$ de la población estudiada a la hora de realizar la acción voluntaria, y es la falta de autoridad frente al usuario, que se produce sobre todo cuando no se tiene la información suficiente y clara para actuar o cuando el profesional a cargo no está cerca para solucionar los posibles inconvenientes que surjan.

La variedad y dispersión de inconvenientes presentados por los Voluntarios Deportivos podemos entenderlo partiendo de la idea en la este recurso humano en grandes eventos deportivos, no requiere un gran esfuerzo por parte de las personas que quieren ayudar, no son actividades continuas ni en espacios ni en tiempo, sino que surgen en la mayoría de las ocasiones cuando existe un acontecimiento deportivo a cubrir, suponiendo casi siempre un compromiso agradable y divertido, en un entorno privilegiado y poco habitual, que llega a cubrir todas las expectativas marcadas por los voluntarios.

3.2. Factores que pueden resultar ser inconvenientes para ser voluntario deportivo, según la opinión de los profesionales.

La labor de los voluntarios tiene que estar en todo momento supervisada y coordinada por un personal profesional remunerado dispuesto para ello. Voluntarios y profesionales ó profesionales y voluntarios
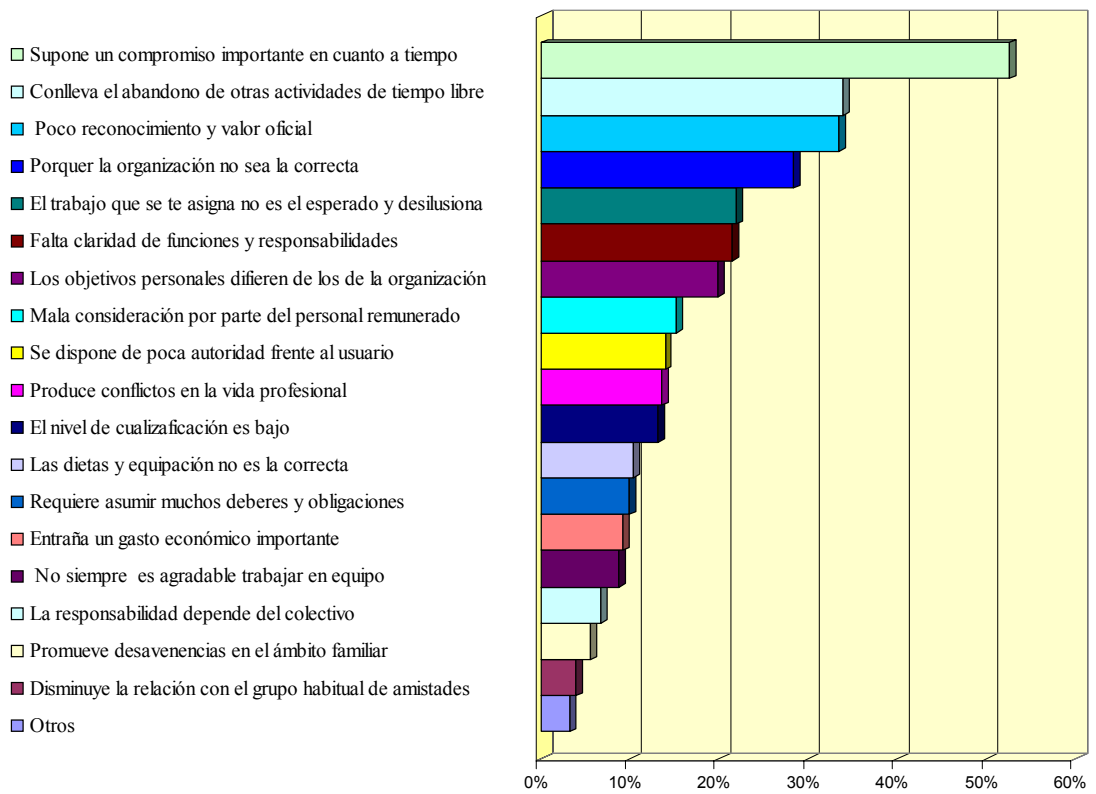

Figura 1. Inconvenientes para realizar actividades de Voluntariado Deportivo, según opinión de los propios voluntarios 
forman un tandem que en muchas ocasiones aparece como indivisible, en la estructura de los recursos humanos de la organización de cualquier evento deportivo. Con respecto a esta situación son los profesionales los que destacan aquellos inconvenientes u obstáculos que, de alguna manera, se producen en una relación obligatoria por parte de la organización y libre por parte de los voluntarios (figura 2).

De esta manera y dentro de los factores que se proponen como inconvenientes, los profesionales señalan como máximo exponente al igual que ocurrió con el grupo de voluntarios, el compromiso que supone en cuanto a tiempo, en un porcentaje del $80 \%$. Este elemento que parte de las propias decisiones personales de cada voluntario, no puede llegar a sorprendernos considerando que vivimos en una sociedad en donde las posibilidades de ocupar nuestro tiempo son muy variadas, siendo el voluntariado una entre muchas.

Otros elementos que según los profesionales pueden resultar un obstáculo para decidir ser voluntario o continuar siéndolo, están relacionados directamente con la organización del evento deportivo, ya que es imposible agradar y conocer a todas las personas que quieren ayudar, percibiendo los voluntarios en algún momento que la organización no sea la más correcta. De hecho los profesionales señalan como un inconveniente que el trabajo que se le asigne no sea el esperado y desilusione (68\%), que la organización no sea la correcta (56\%) y que los objetivos y motivaciones personales no se adapten a los de la organización (52\%).

Relacionándolo igualmente con la organización de la actividad, podría entenderse que si las dietas y equipación que se proporcionan no es la correcta $(36 \%)$ sería un factor a tener en consideración para la no participación en actividades de voluntariado deportivo, además de no tener claro las funciones y responsabilidades de cada persona (32\%).

Otro grupo de inconvenientes que son señalados por el grupo de profesionales con porcentajes importantes, tienen que ver con el entorno social que rodea las actuaciones. El poco reconocimiento social y valor oficial de las tareas de voluntariado (48\%), la mala consideración por parte del personal remunerado (28\%) y el disponer de poca autoridad frente al usuario (24\%) hacen que surja la idea de que son los propios profesionales quienes asumen el poco peso que tiene la labor voluntaria en determinados momentos.

3.3. Comparativa de las opiniones de los Voluntarios Deportivos y profesionales sobre los factores que pueden ser considerados inconvenientes para colaborar como voluntarios deportivos.
Los datos que hemos establecido hasta el momento, no adquieren su verdadero significado hasta no observar en una comparativa los resultados obtenidos en los dos grupos estudiados, que como ya hemos insistido anteriormente están destinados a convivir y como consecuencia a conocerse y reconocerse.

Aparece una gran disparidad porcentual entre las opiniones de los dos colectivos en torno a los posibles inconvenientes que puede producir la colaboración como Voluntario Deportivo, siendo llamativo el hecho de que los profesionales perciben o detectan más inconvenientes de lo que los propios voluntarios indican (figura 3).

Destacamos inicialmente, entre el conjunto de problemas personales (Domingo, 1996; Aguilera Luna, 2003 y Cortés García y Matías Cabrera, 2004), la circunstancia de que supone un compromiso importante en cuanto a tiempo. Ambos grupos de sujetos la señalan con el porcentaje más alto pero son los profesionales quienes mayoritariamente indican esta opción (superando en treinta y una unidades porcentuales al otro colectivo), pudiéndose deducir que el trabajo que realizan los profesionales es muy favorecedor para la puesta en marcha del grupo de voluntarios. Este resultado, por otra parte no sorprende, ya que, como afirman Robinson y Gobey, 1999 y VV.AA., 2004b, el elemento tiempo en las sociedades desarrolladas es un bien que escasea y al que se le procura dar un valor importante y una utilización adecuada a las necesidades personales y profesionales de cada uno.

Los profesionales que trabajan con grupos de Voluntarios Deportivos observan que los obstáculos para realizar tareas de voluntariado pueden surgir fundamentalmente de la propia organización (Domingo, 1996; Aguilera Luna, 2003 y Cortés García y Matías Cabrera, 2004), porque ella no sea capaz de cubrir todas las expectativas previstas por los colaboradores. Pero esa preocupación está lejos de la opinión del grupo de voluntarios, que más allá del problema de falta de tiempo libre personal (señalada por más de la mitad de ellos) no encuentran dificultades (los porcentajes no superan en ningún caso el tercio de la población) para poder colaborar en actividades de Voluntariado Deportivo, adaptándose a cualquier labor o tarea que la organización les asignen.

Quizás esta idea venga precedida de la falta de formación de los profesionales hacia los voluntarios, intentando con ello decir que son dos grupos de personas que trabajan bajo el objetivo común de hacer de la organización del evento deportivo a cubrir, un éxito de todos, pero con objetivos secundarios muy diversos y diferentes aunque casi nunca contrapuestos.

La relación de mutua cooperación, se entrelaza constante y necesariamente con conceptos de complementariedad y subordinación, sin

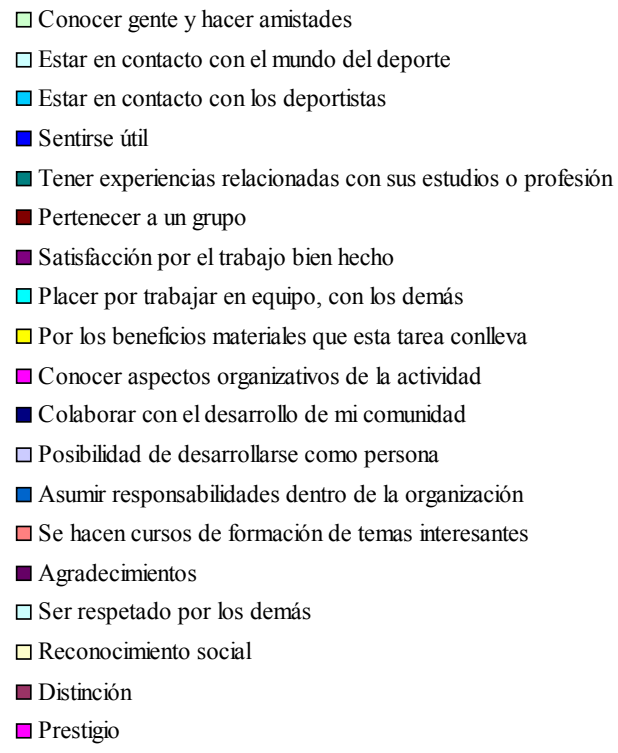

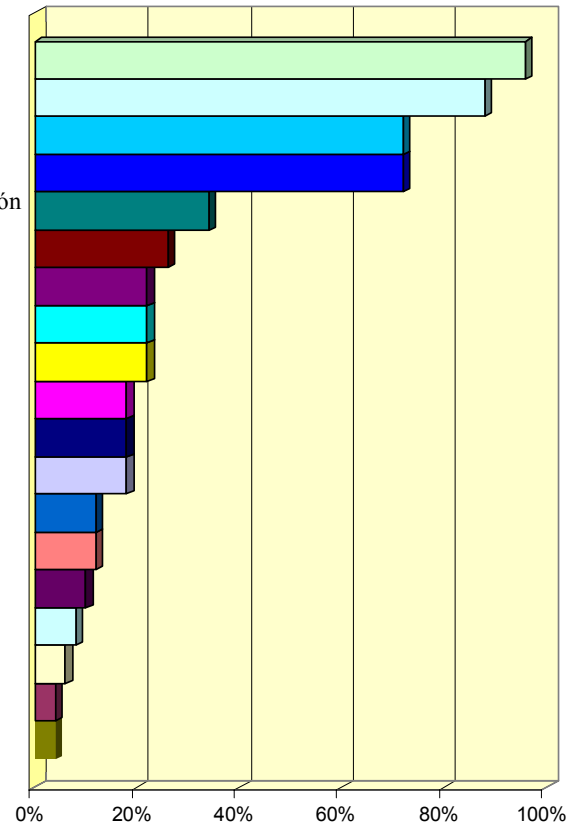

Figura 2. Inconvenientes para realizar actividades de Voluntariado Deportivo, según la opinión del personal profesional remunerado. 
que con ello tenga que suponer una confrontación directa entre los dos grupos de personas. Parece ser por ello necesario, una formación completa y correcta del grupo de profesionales hacia la utilización del recurso del voluntariado.

En efecto, siguen siendo los profesionales, treinta y ocho unidades porcentuales más que los voluntarios, quienes remarcan con mayor fuerza el obstáculo que puede llegar a suponer que el trabajo que se le asigna no es el esperado y desilusione, dando con ello a entender los profesionales, que son conscientes de la dificultad de cubrir todas las necesidades y expectativas de los voluntarios, a pesar de intentarlo por el bien y el éxito de la organización. Podemos observar que los voluntarios son conocedores de que su labor no es sólo hacer una determinada tarea, sino que el entorno, la gente que se llega a conocer, las vivencias que se producen... cubren la parte más importante de la acción voluntaria.

Bajo la misma idea anterior podemos destacar los desacuerdos entre los dos grupos estudiados en torno al obstáculo que puede suponer que la organización no sea la correcta, donde los profesionales lo señalan superando en veintiocho unidades porcentuales a los voluntarios, o el caso del inconveniente que puede llegar a ser que los objetivos y motivaciones personales de los voluntarios no se adapten a los de la organización, donde asciende hasta el treinta y dos por ciento la diferencia de los profesiones sobre los voluntarios. Podemos llegar a pensar que los profesionales perciben desde el interior de la organización que ésta no es siempre la más correcta, en tanto que para los voluntarios la organización es sólo una parte de su labor que no puede empañar ni trabar una actuación solidaria y altruista, en donde lo importante es participar y en donde «se llega a recibir más que a dar».

El resto de obstáculos son percibidos minoritariamente por ambos colectivos (siempre por debajo del cincuenta por ciento). Llama la atención que, en relación a cuestiones materiales como las dietas y la equipación que se les proporciona, así como al posible gasto económico que le supone a la persona que presta sus servicios voluntariamente, sean igualmente los profesionales los que más destaquen (superando en veintiséis y siete unidades porcentuales respectivamente).

Siguiendo con los inconvenientes procedentes de la organización, es loable que sean los propios profesionales remunerados los que reconozcan, en mayor medida (por la responsabilidad que deben asumir), como posibles la falta de claridad de funciones y responsabilidades (diez unidades porcentuales más) y la circunstancia del reconocimiento a la labor del Voluntariado Deportivo por parte de los profesionales (quince puntos) y los usuarios (diez puntos) a los que se asiste, confirmando las afirmaciones realizadas por Nichols y King (1998) y Heinemann (1999). Vuelven a dar los voluntarios el mismo mensaje ante la organización, apostando por una labor desinteresada y altruista, olvidando las posibles incidencias negativas y agarrándose a la posibilidad de ayudar pasando un rato agradable, percibiendo lo positivo de su labor.

A partir de esta idea hay que decir que sólo surge un obstáculo que los Voluntarios perciben con mayor insistencia que los profesionales. Se trata de la mala consideración por parte del personal remunerado hacia los voluntarios. Quizás sea por las exigencias de los profesionales hacia su labor, que por otra parte se produce dentro de su espacio y tiempo laboral, en tanto que la actuación de los colaboradores se realiza como alternativa en su tiempo libre, no considerando en ella rasgos de un trabajo profesional, como adelantaban Collozi y Bassi (1995).

La convivencia de espacios y tiempos contrapuestos de estos dos grupos de personas, puede producir y de hecho produce uno de los más importantes conflictos entre organización y voluntarios si no se analiza y actúa correctamente sobre ello. De todo ello podemos deducir que a pesar de que el trabajo voluntario tiene su raíz en el altruismo y el desinterés, el egoísmo de la naturaleza humana siempre aparece. Los voluntarios buscan realizar tareas que de alguna forma a ellos les interese desde el punto de vista personal o profesional, y que su labor, a pesar de tener un origen filántropo, sea valorada por el entorno al que se beneficia, de no ser esto así podría producir el abandono en las actividades de Voluntariado Deportivo.

Por último es de destacar, por los bajos porcentajes tanto de voluntariado como de personal remunerado que los avalan, las cuestiones relacionadas con el entorno que rodea a este mundo: las repercusiones que produce el trabajo voluntario en las relaciones familiares y laborales.

\section{Conclusiones.}

Entre las conclusiones que hacen referencia a los posibles conflictos y adversidades que surgen en la utilización del recurso del Voluntariado Deportivo en grandes eventos señalamos:

- La percepción porcentual de inconvenientes es mayor entre los profesionales remunerados que entre el voluntariado deportivo, el cual parece mostrarse menos exigente en consecuencia con la labor altruista que consideran conllevan estas acciones.

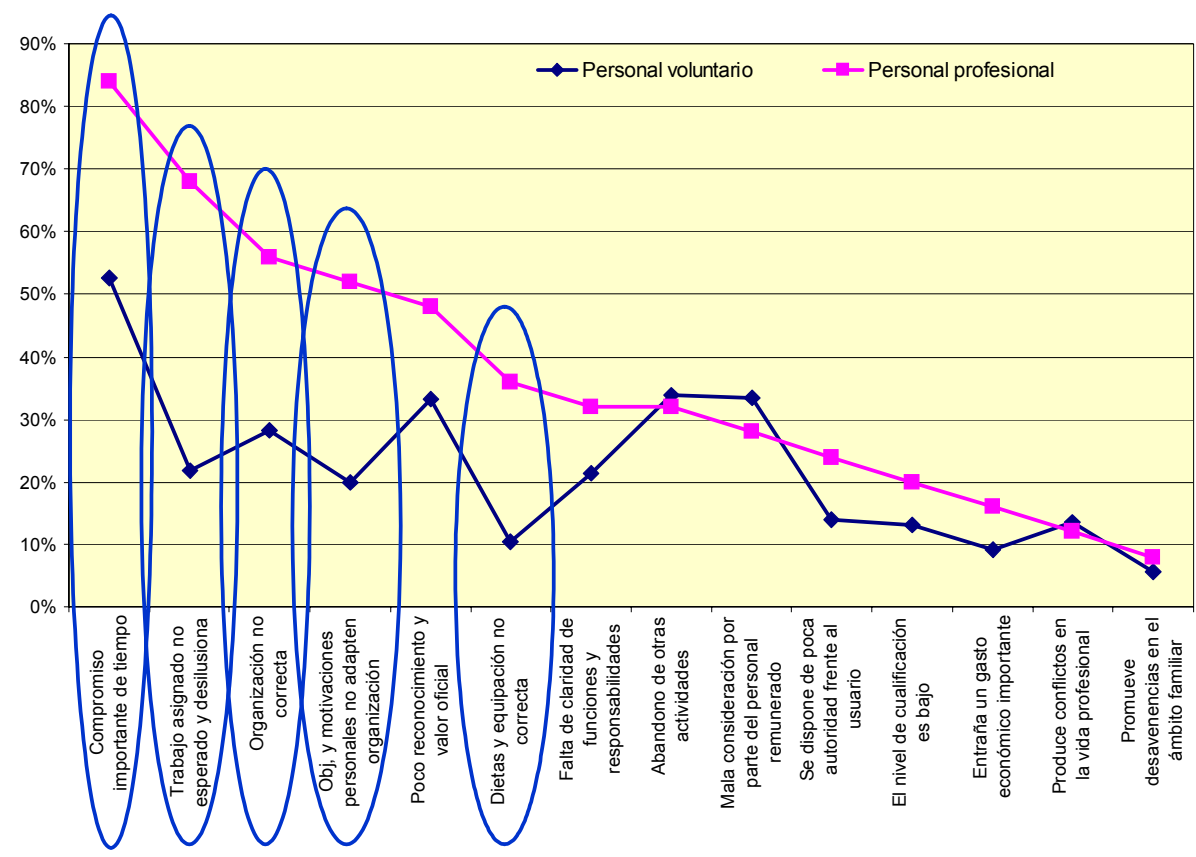

Figura 3. Comparativa entre la opinión de los voluntarios y la de los profesionales Remunerados sobre los inconvenientes de la labor voluntaria 
- Entre el voluntariado deportivo solo es refrendado por más de la mitad de la población el inconveniente de suponer un compromiso importante en cuanto a tiempo, siendo identificado mayoritariamente por los profesionales remunerados.

- El reconocimiento, por parte de los profesionales remunerados, de que los mayores obstáculos pueden provenir de aspectos relacionados fundamentalmente con la organización, al no ser capaz de responder a las expectativas del voluntariado, desvelan la necesidad, por parte de las organizaciones, de ser conocedores de cuál es la realidad de la labor voluntaria en este sector y tener una mayor formación hacia este sector de recursos humanos.

- Los resultados muestran que los dos colectivos de personas trabajan bajo el objetivo común de hacer de la organización del evento deportivo a cubrir, un éxito de todos, pero con objetivos secundarios muy diversos y diferentes, aunque casi nunca contrapuestos. - El obstáculo que aparece más resaltado por el voluntariado deportivo tiene que ver con la mala consideración por parte del personal remunerado, mostrando las reticencias existentes entre dos situaciones espaciales y temporales diferentes, la ocupación de su tiempo libre, entre los primeros, tal vez con cierta pretensiones de que les sea útil para su futuro laboral y la implicación propiamente laboral, entre los segundos.

Para finalizary como idea final decir que, el mundo del Voluntariado Deportivo tiene luces pero también sombras que hay que iluminar, para no terminar haciendo de este instrumento un mero número de puestos y tareas a cubrir. No llegar a equivocar la cantidad con la calidad de un recurso humano cada vez más necesario, por muchos y controvertidos motivos. La politización de este tipo de actividad, la precariedad con la que en muchas ocasiones se prestan las colaboraciones o las exigencias por parte de la organización hace que no siempre sean entendidas o valoradas en sus diferentes dimensiones.

\section{Bibliografía.}

Abadía Naudi, S. (2004). El perfil del voluntario de los «X FINA» campeonatos del mundo de natación Barcelona 03. Apunts, educación fisica y deportes, 77, 79-84.

Aguilera Luna, J. L. (2003). ¿Crisis o auge del voluntariado?. Revista Summa de Voluntades, $\mathrm{n}^{\circ}$ 2, mayo 2003. www.sumadevoluntades.org (consulta: 10 septiembre 2003).

Boletín Oficial de la Junta de Andalucía n 84 de 2001, Ley 7/2001, de 12 de julio, del Voluntariado, 12.686-12.693.

Chavarria, X. (2005). Vivir de la actividad física. Tándem, didáctica de la Educación Física, 19, 7-17

Colás, P. (1994). La metodología cualitativa en educación. Aportaciones científicas a la educación. Revista Bordón, 46 (4), 407-423.

Colozzi, I. y Bassi,A. (1995). Una soliedarietá efficiente. Il terzo settore ele organización di voluntariato. Roma: La nueva Italia Científica. Cortés Alcalá, L.; Hernán Montalbán, Mª J. y López Maderuelo, O (1997). Las organizaciones de voluntariado en España. Madrid: Plataforma para la promoción del voluntariado en España.

Cortés García, G. y Matías Cabrera, A. (2004). Material didáctico de voluntariado en Andalucía. Segunda Edición. Málaga: Plataforma andaluza de voluntariado.

Domingo, A. (1996). ¿Voluntarios? No, gracias: clarificación ética de la acción voluntaria. Documentación Social (104), 27-38.

Handy, C. (1988). Understanding voluntary organizations. London: Penguin.

Heinemann, K (1999). Sociología de las organizaciones voluntarias. El ejemplo del club deportivo. Valencia: Tirant lo blanch.

Heinemann, K (2004). ¿Es sostenible el deporte actual?. Un análisis desde la perspectiva ética. Apunts, educación fisica y deportes, 78, 10-18

Marbán Gallego, V. y Rodríguez Cabrero. (2001). «El voluntariado: prácticas sociales e impactos económicos». Revista del Ministerio de Trabajo y Asuntos Sociales, Madrid.

Martínez del Castillo, J. (1997). «El proceso de profesionalización de los recursos humanos de las asociaciones deportivas en España». Revista Apunts, (49), 63-83.

Nichols, G. y King, L. (1998). «Research for the Guide Association; problems and solutions». Voluntary Action, (1), 21-32.

Pérez Pérez, I. (2004). La figura del voluntariado en la organización de actividades fisico-deportivas. Características e incidencia en la estructura de los recursos humanos. Tesis Doctoral. Universidad de Granada.

Roca i Balasch, J. (2003). Ciencias y profesiones en la actividad fisica y el deporte. Apunts, educación fisica y deportes, 74, 60-66.

Robinson, R. y Godbye, G. (1999). Time for life: the surprising ways Americans use their time. Pennsylvania: Pennsylvania State University Press.

Vaca Escribano, M.J. (2002). Reflexiones entorno a las competencias profesionales solicitadas a los maestros de Eduación Física con la implantación de la LOGSE. Retos, nuevas tendencias en educación fisica, deporte y recreación, 3, 13-15.

VV.AA. (2004a). Plan General del Deporte en Andalucía. Junta de Andalucía, Consejería de Turismo y Deporte.

VV.AA. (2004b). Estudio sobre el voluntariado social de Andalucía. Junta de Andalucía, Consejería de Asuntos Sociales.

Weisbrod, B.A.(1978). The voluntary non-profitsector. Massachusetts: Lexington Books.

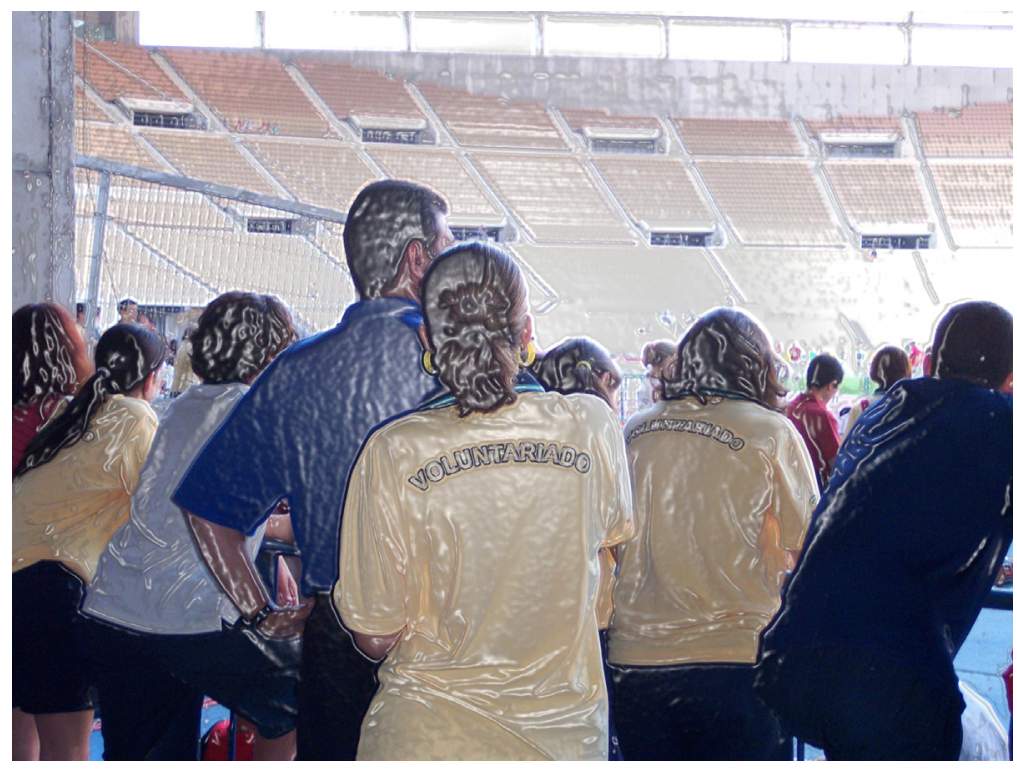

